\title{
Peptidomic screening of bitter and nonbitter casein hydrolysate fractions for insulinogenic peptides
}

\author{
Niamh M. Murray, ${ }^{*}$ Dolores O’Riordan, ${ }^{*}$ Jean-Christophe Jacquier, ${ }^{*}$ Michael O’Sullivan, ${ }^{*}$ Thérèse A. Holton, $\dagger$ \\ Kieran Wynne,† Randall C. Robinson, $\ddagger$ Daniela Barile, $\ddagger$ Søren D. Nielsen, $\S$ and David C. Dallas $\S^{1}$ \\ *Food for Health Ireland, UCD Institute of Food and Health, and \\ †UCD Conway Institute of Biomolecular and Biomedical Research, University College Dublin, Belfield, Dublin 4, Ireland \\ ‡Department of Food Science and Technology, University of California, Davis 95616 \\ $\S N u t r i t i o n$ Program, School of Biological and Population Health Sciences, College of Public Health and Human Sciences, Oregon State University, \\ Corvallis 97331
}

\begin{abstract}
Sodium caseinate hydrolysates $(\mathrm{NaCaH})$ contain biologically active peptides that can positively influence human health. However, their intense bitterness hinders their inclusion in food products. To our knowledge, no studies have investigated whether a correlation between bitterness and bioactivity exists in $\mathrm{NaCaH}$, so it is not yet known what effect selective removal of bitterness has on $\mathrm{NaCaH}$ bioactivity. A deeper understanding of the physicochemical characteristics affecting both bitterness and bioactivity is therefore needed. The aim of this study was to use in silico analysis to elucidate the relationship between bitterness and bioactivity of the insulinogenic $\mathrm{NaCaH}$. The $\mathrm{NaCaH}$ fractions were generated by membrane filtration and flash chromatography and were subsequently evaluated for bitterness by a sensory panel. In this present study, peptidomic and bioinformatic processing of these $\mathrm{NaCaH}$ fractions allowed for the identification of insulinogenic peptides as well as other literature-identified peptides in each of the fractions. The results showed that the most bitter fraction contained the highest abundance of insulinogenic peptides, whereas another bitter fraction contained the highest abundance of other literature-identified bioactive peptides exhibiting angiotensin-converting enzyme-inhibition activity. Although some bioactive peptides were identified in the least bitter fractions, the abundance of these peptides was very low. These observations show a correlation between bitter taste and bioactivity, highlighting potential complications in removing bitterness while maintaining bioactivity. However, as the most bitter fraction contained the highest abundance of insulinogenic peptides, there is potential for using a lower dose of this enriched bio-
\end{abstract}

Received September 18, 2017.

Accepted December 14, 2017.

${ }^{1}$ Corresponding author: dave.dallas@oregonstate.edu active fraction to exert health benefits. The second most bitter fraction contained a very low abundance of insulinogenic peptides and other bioactive peptides. Therefore, removal of this fraction could reduce the Na$\mathrm{CaH}$ product's bitterness without significantly altering overall bioactive potential.

Key words: casein hydrolysate, bioactivity, bitterness, insulinogenic peptide, peptidomic approach

\section{INTRODUCTION}

The increase in nutrition-related diseases has led to increased interest in the use of functional food products for disease prevention. Dairy protein hydrolysates (DPH) have great potential in this market due to the bioactive peptides found in $\mathrm{DPH}$, which have a range of sequence-specific actions (Saavedra et al., 2013), including anti-inflammatory (Hernández-Ledesma et al., 2014), immunomodulatory (Hernández-Ledesma et al., 2014), angiotensin-converting enzyme (ACE)-inhibitory (Nongonierma and FitzGerald, 2015), opioid (Nongonierma and FitzGerald, 2015), antioxidant (Power et al., 2013; Irshad et al., 2015; Nongonierma and FitzGerald, 2015), antimicrobial, dipeptidyl peptidase-4 inhibitory (DPP-IV), antithrombotic (Nongonierma and FitzGerald, 2015), and antiproliferative (Hernández-Ledesma et al., 2014) actions. A sodium caseinate hydrolysate $(\mathbf{N a C a H})$ has been reported to reduce blood glucose levels and increase insulin levels in healthy participants (aged 40-65 yr with a BMI $>25$ $\mathrm{kg} / \mathrm{m}^{2}$ ) when a $12 \mathrm{~g}$ dose of $\mathrm{NaCaH}$ was consumed along with a carbohydrate meal (Brennan et al., 2015). Eleven insulinogenic peptides were responsible for exerting the insulinogenic effects in the $\mathrm{NaCaH}$; their sequences have been identified and published (Brennan et al., 2015).

Despite the strong scientific and commercial interest in this insulinogenic $\mathrm{NaCaH}$ (Brennan et al., 2015), its incorporation into foods remains a challenge due 
to its intense bitter taste. The bitterness of DPH is determined by several factors, including the molecular weight and hydrophobic profile of the peptides present in the DPH (Leksrisompong et al., 2010; Newman et al., 2014; Murray et al., 2017), the type of enzyme used to hydrolyze the protein (O'Sullivan et al., 2017), and the hydrolysis processing conditions (e.g., pH, temperature, degree of hydrolysis, enzyme:substrate ratio; Nongonierma and FitzGerald, 2017; O'Sullivan et al., 2017). The sequence of $\mathrm{AA}$ in a peptide chain can also influence peptide bitterness (Ishibashi et al., 1987a, b), but as DPH contain hundreds of peptides, the task of tasting synthesized peptides to determine the peptide(s) responsible for imparting a bitter taste is a long and difficult process. Therefore, identifying the key factors responsible for DPH bitterness is an extremely challenging task. Several techniques can be used to predict or identify bitterness in DPH. These include peptidomic characterization, for the prediction of bitter peptides using database searching (Iwaniak et al., 2016), and sensory-guided fractionation (Toelstede and Hofmann, 2008; Karametsi et al., 2014; Liu et al., 2014). Sensory-guided fractionation involves fractionating DPH into less-complex fractions (in terms of peptide composition) and then evaluating the fractions for bitterness. This approach was used by Murray et al. (2017) to identify the fractions or physicochemical characteristics most responsible for bitterness in the $\mathrm{NaCaH}$ described by Brennan et al. (2015).

As consumer acceptance of a product depends heavily on taste (Udenigwe, 2014), developing DPH products with reduced bitterness and maintained bioactivity is desirable; such a nuanced approach to product development requires a deeper understanding of the physicochemical characteristics of both bitterness and bioactivity in DPH. However, to our knowledge no studies have aimed to elucidate this relationship. Considering that both bitterness and bioactive compounds are mediated through G protein-coupled receptors (Regard et al., 2007; Behrens and Meyerhof, 2011), a correlation may not be too surprising. Testing the bioactivity of $\mathrm{NaCaH}$ fractions, obtained from sensory-directed fractionation, may elucidate the relationship between $\mathrm{NaCaH}$ bitterness and bioactivity.

Therefore, the objective of the present study was to elucidate the relationship between bitterness and bioactivity of the insulinogenic $\mathrm{NaCaH}$ described by Brennan et al. (2015). To achieve this objective, we used peptidomics to establish the sequences of the many peptides present in the $\mathrm{NaCaH}$ and each of its subfractions, which were generated as described by Murray et al. (2017). Bioinformatic analysis of the peptidomic data was then used to identify which fraction(s) contained the most bioactive peptides through sequence search- ing against databases such as BIOPEP (Minkiewicz et al., 2008) and the Milk Bioactive Peptide Database (Nielsen et al., 2017).

\section{MATERIALS AND METHOD}

\section{Chemicals and Materials}

The NaCaH parent product (Brennan et al., 2015) was provided by Moorepark Technology Ltd. (Fermoy, Co. Cork, Ireland). The $\mathrm{NaCaH}$ fractions $[<3-\mathrm{kDa}$ permeate and 5, 10, 30, and 50\% ethanol reverse-phase (RP) flash chromatography fractions] were obtained as outlined in Murray et al. (2017). Briefly, the $\mathrm{NaCaH}$ was first fractioned by membrane filtration to yield a low-molecular-weight fraction $(<3-\mathrm{kDa})$, which was subsequently further fractionated by RP flash chromatography to produce fractions soluble in $5,10,30$, and $50 \%$ ethanol. For peptide quantification of the $\mathrm{NaCaH}$ fractions, a Pierce bicinchoninic acid protein assay kit was obtained from Thermo Fisher Scientific (Waltham, MA). The solvents used for the MS characterization of the $\mathrm{NaCaH}$ fractions were HPLC-grade formic acid (FA), trifluoroacetic acid (TFA), and acetonitrile (ACN; Sigma-Aldrich, St. Louis, MO).

\section{Peptide Analysis by MS}

All samples $(\mathrm{NaCaH},<3-\mathrm{kDa}$ permeate, and the 5 , 10,30 , and $50 \%$ ethanol flash chromatography fractions) were analyzed by a quadrupole time-of-flight (Q-TOF) MS to identify peptides with an AA chain length of $\leq 5$ using de novo sequencing and an Orbitrap MS (Thermo Fisher Scientific) to identify peptides $>5$ AA in length using database searching.

Orbitrap Analysis. Liquid chromatography separation was performed on a Waters Nano Acquity UHPLC (Waters Corp., Milford, MA) with a Proxeon nanospray source. All fractions were solubilized $(1 \mathrm{mg} / \mathrm{mL})$ in $2 \%$ CAN and $0.1 \%$ TFA in nanopure water. As the 30 and $50 \%$ ethanol fractions were poorly soluble in $2 \% \mathrm{ACN}$ and $0.1 \%$ TFA, solubilization was aided by sonication (Ultrasonic processor Q55, Qsonica, Newtown, CT) at $60 \%$ amplitude for $1.5 \mathrm{~min}$. Two micrograms of each sample was first loaded onto a $100 \mu \mathrm{m} \times 25 \mathrm{~mm}$ Magic C18 $100 \AA 5 \mathrm{U}$ reverse-phase trap for enrichment and online desalting and then onto a $75 \mu \mathrm{m} \times 150 \mathrm{~mm}$

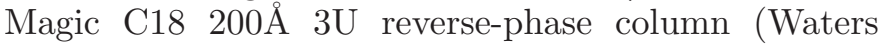
Corp.) for analytical separation. Peptides were eluted using a gradient of $0.1 \%$ FA (A) and $100 \%$ ACN (B) with a flow rate of $300 \mathrm{~nL} / \mathrm{min}$. A 60 -min gradient was run with 5 to $35 \%$ solvent B over $50 \mathrm{~min}, 35$ to $80 \%$ solvent B over $3 \mathrm{~min}, 80 \%$ solvent B for $1 \mathrm{~min}, 80$ to $5 \%$ solvent B over $1 \mathrm{~min}$, and finally held at $5 \%$ solvent 
B for 5 min. Each sample run was followed by a 30-min column wash.

Mass spectra were collected on an Orbitrap Q-Exactive Plus MS (Thermo Fisher Scientific) as described previously (Dallas et al., 2016). The samples were analyzed a single time by the Orbitrap.

Spectra were analyzed by database searching using X!Tandem (Craig and Beavis, 2004) against a bovine milk library according to a previously described procedure (Dallas et al., 2013, 2014). Identified peptides were accepted if e-values were $\leq 0.01$, corresponding to a confidence level of $99 \%$. Peptide mass tolerance was $20 \mathrm{ppm}$ for both precursor and fragment ions. No complete (required) modifications were applied. Potential modifications allowed included methionine oxidation in the initial search and serine and threonine phosphorylation in a refinement round. A nonspecific cleavage $([\mathrm{X}] \mid[\mathrm{X}]$, where $\mathrm{X}$ is any $\mathrm{AA})$ was used to search against the protein sequences. Because the instrument did not always select the monoisotopic ion for tandem fragmentation, isotope errors were allowed (allowing up to one C13).

The output .xml libraries of identified peptides from $\mathrm{X}$ !Tandem analysis was uploaded into Skyline (Schilling et al., 2012) to create the spectral libraries. The spectral libraries were searched against the raw data files from the Orbitrap Q-Exactive Plus MS to extract the peak areas for each peptide using settings as described previously with few modifications (Dallas et al., 2016). Briefly, "precursor mass" was set to the monoisotopic ion, and "ion types" was set to precursor only. Match tolerance was set to 0.5 mass:charge ratio $(\boldsymbol{m} / \boldsymbol{z})$. The acquisition window was set between 150 and $1,600 \mathrm{~m} / z$, with a tolerance of $0.055 \mathrm{~m} / \mathrm{z}$. Retention time filtering was set to within $1 \mathrm{~min}$ of MS/MS identification.

$\boldsymbol{Q}$-TOF Analysis. All fractions were solubilized (1 $\mathrm{mg} / \mathrm{mL}$ ) in $2 \% \mathrm{ACN}$ as outlined in the section above. The samples were analyzed on an Agilent Technologies (Santa Clara, CA) nano-LC-chip Q-TOF MS/MS. The bicinchoninic acid assay was used to determine the peptide concentration for the samples. Appropriate dilutions were made to load $0.1 \mu \mathrm{g}$ of sample onto the Agilent chip C18 column at a flow rate of $0.3 \mu \mathrm{L} /$ min. Peptides were first loaded onto the enrichment column and then onto a $75 \mu \mathrm{m} \times 150 \mathrm{~mm}$ separation column packed with Zorbax 300SB-C18 5 - $\mu \mathrm{m}$ material for analytical separation. The gradient elution solvents were $3 \% \mathrm{ACN}$ and $0.1 \% \mathrm{FA}$ (solvent $\mathrm{A}$ ) and $89.9 \%$ ACN and $0.1 \%$ FA (solvent B). The 65-min gradient was increased from 2.3 to $8 \%$ solvent B from 0.1 to 2 min, 8 to $37 \%$ solvent B from 2 to $40 \mathrm{~min}, 37$ to $48 \%$ solvent B from 40 to $45 \mathrm{~min}$, and 48 to $100 \%$ solvent B from 45 to $45.1 \mathrm{~min}$; held at $100 \%$ solvent B from 45.1 to $50 \mathrm{~min}$; and then equilibrated with solvent $\mathrm{A}$ for the remainder of the run. The capillary pump was set to $4 \mu \mathrm{L} / \mathrm{min}$ and $0 \% \mathrm{~B}$ throughout the analysis. Ion polarity was set to positive. Data were collected in centroid mode. The drying gas was $325^{\circ} \mathrm{C}$ with a flow rate of $5 \mathrm{~L} / \mathrm{min}$. The spectral acquisition rate was set to $5 \mathrm{spectra} / \mathrm{s}$ for MS. For MS/MS scans, a variable scan speed was used with a target total ion abundance of 25,000 counts/spectrum and a minimum rate of 1.3 spectra/s, with up to $10 \mathrm{MS} / \mathrm{MS}$ spectra after each MS spectrum. The MS spectra were acquired from 70 to $1,000 \mathrm{~m} / z$, and the MS/MS spectra were acquired from 20 to $1,000 \mathrm{~m} / z$. Automated precursor selection based on abundance was used with active precursor exclusion after 2 times selected for $0.5 \mathrm{~min}$. The precursor isolation window for MS/MS analysis was $1.3 \mathrm{~m} / \mathrm{z}$. The collision energy was set by the formula slope $\times(\mathrm{m} / z) / 100$ + offset, with slope $=3$ and offset $=2$. Mass calibration was performed during data acquisition based on infused calibrant ions with masses of 121.050873 and 922.009798 Da (from product no. G1969-85001; Agilent Technologies). Each sample run was followed by a 60-min column wash. The Q-TOF analysis was carried out in triplicate for each of the samples.

Raw Q-TOF spectral data were processed using the de novo sequencing module of PEAKS (Ma et al., 2003). The search algorithm used a false discovery rate of $1 \%$. Only peptides with an average local confidence of $70 \%$ or above were retained. Average local confidence is the sum of the confidence level (0-1) that each AA in the sequence is correct divided by the number of AA in the sequence. The parent mass error tolerance was $20 \mathrm{ppm}$, and the fragment mass error tolerance was $0.5 \mathrm{Da}$. The enzyme pattern selected was "none" (nonspecific). Following the approach of Holton et al. (2014), peptide data from triplicate Q-TOF sample runs were condensed to a single consensus, where only peptides detected in a minimum of 2 MS replicates were retained. The relative abundances of peptides identified by PEAKS were extracted from the MS data as peak areas using Agilent MassHunter Profinder B.06.00 (Agilent Technologies). Chromatographic peaks for the peptides were identified by mass and retention time, with a $10-\mathrm{ppm} \mathrm{m} / z$ error tolerance and a 2.5-min retention time tolerance. The search identified all peptides as hydrogen ion adducts with a maximum allowed charge of $2+$ and a minimum chromatographic peak height of 400 ion counts.

\section{Bioinformatics}

The peptides identified by the Orbitrap and Q-TOF MS data were searched against a local version of the BIOPEP (Minkiewicz et al., 2008) bioactive peptide database and the Milk Bioactive Peptide Database (Nielsen et al., 2017) to identify known bioactive pep- 
tides; the fractions were also screened for the insulinogenic peptides (Brennan et al., 2015). For all peptide searches, only exact matches were considered.

\section{RESULTS AND DISCUSSION}

The bitterness of the $\mathrm{NaCaH}$ subfractions $(<3-\mathrm{kDa}$ permeate and flash chromatography fractions: 5, 10, 30 , and $50 \%$ ethanol) was determined by a sensory panel $(\mathrm{n}=12)$, as described by Murray et al. (2017). The results showed an increase in bitterness with an increase in hydrophobicity of the low-molecular-weight fraction $(<3-\mathrm{kDa}$ permeate), with an exception of the most hydrophobic fraction (50\% ethanol), which had low bitterness scores. Based on a 10-cm structured line scale, the bitterness scores assigned by a sensory panel were as follows: the parent $\mathrm{NaCaH}=4.4$, the $<3-\mathrm{kDa}$ permeate $=6.4,5 \%$ ethanol $=0.7,10 \%$ ethanol $=6.7$, $30 \%$ ethanol $=8.5$, and $50 \%$ etOH $=1.5$. This present study aimed to investigate whether a similar trend or correlation was observed with the presence of bioactive peptides in each of the $\mathrm{NaCaH}$ subfractions.

\section{Q-TOF and Orbitrap-Identified Peptides}

Orbitrap analysis was used to identify peptide sequences of $>5 \mathrm{AA}$ in length in the $\mathrm{NaCaH}$ and $\mathrm{NaCaH}$ subfractions. The Orbitrap MS data identified between 191 and 701 peptides in the $\mathrm{NaCaH}$ fractions. The $<3$ $\mathrm{kDa}$ permeate contained the highest Orbitrap ( $>5 \mathrm{AA}$ ) peptide count, followed by the $30 \%$ ethanol fraction, the $\mathrm{NaCaH}$, and the 50,10 , and $5 \%$ et $\mathrm{OH}$ flash chromatography fractions (Figure 1A). The $<3$-kDa permeate also contained the highest total peptide abundance, followed by the $30 \%$ ethanol, the $\mathrm{NaCaH}$, and the 10,50 , and 5\% ethanol fractions (Figure 1B). Despite injecting equal concentrations of extracted peptides (based on weight), the abundance of Orbitrap-identified peptides in the 5 and $50 \%$ ethanol fractions was low. This could be due to a high content of salts and free AA or shorter chain peptides ( $<5 \mathrm{AA}$ in length) that could not be detected or identified by the Orbitrap gradient or $\mathrm{X}$ !Tandem software. In addition, the peptides eluted in these fractions may have had lower ionization efficiency than peptides in the other fractions, resulting in a lower ion abundance.

We used Q-TOF and PEAKS analysis to identity peptide sequences 1 to $5 \mathrm{AA}$ long in each fraction (Figure $1 \mathrm{C})$. The use of an MS scan window of $<1,000 \mathrm{~m} / z$ targeted the Q-TOF analysis to mostly smaller peptides. MassHunter Profinder B.06.00 (Agilent Technologies) was then used to calculate the abundance of these peptides within each of the fractions. In numerous cases it was not possible to determine the exact order of AA within these smaller peptides from the MS/MS data. We have therefore presented all isomeric possibilities for each peptide: FALP and AFLP, YVPL and VLPY, LHPF and LHML, LVPVP and VLPVP, AFLPQ and FALPQ, LYGYL and YLGYL, ELEEL and LEEEL, LNFLK and QVFLK, SFQLF and YAQLF, MTPLW and TMPLW, DLLFK/LDFLK/LDLFK triad (the peak areas from LDFLK were used), and LPLQY/LYPLQ/YLPLQ triad (the peak areas from LPLQY were used). Between 16 and 68 unique Q-TOF peptides were identified in the individual $\mathrm{NaCaH}$ fractions. Again, the $<3$-kDa permeate contained the highest count of unique peptides, followed by the $\mathrm{NaCaH}$, the $30 \%$ ethanol fraction (which contained equal counts of peptides), and the 5, 10, and 50\% ethanol fractions, respectively (Figure 1C). However, it was the 5 and $10 \%$ ethanol fractions that contained by far the highest abundance of shorter chain peptides (Figure 1D). This may account for the low abundance of Orbitrap-identified peptides in the $5 \%$ ethanol fraction. Peptide interaction with an RP stationary phase can be influenced by its AA chain length; generally, larger peptides are more hydrophobic than smaller peptides and thus may have increased interaction or binding ability to a C18 stationary phase (Ciborowski and Silberring, 2016). The Q-TOF-identified peptides of $<5 \mathrm{AA}$ in length may have had limited interaction with the $\mathrm{C} 18$ stationary phase and were thus eluted in the most hydrophilic fraction ( $5 \%$ ethanol).

The most abundant proteins in bovine milk are $\alpha_{\mathrm{S}^{-}}$ and $\beta$-CN (Chatchatee et al., 2001), and peptides derived from these proteins have been shown to be bitter (Singh et al., 2003). The $<3-\mathrm{kDa}$ permeate contained the highest abundance of peptides derived from $\alpha_{\mathrm{S1}^{-}}$ $\mathrm{CN}$, followed by the $30 \%$ ethanol fraction, the $\mathrm{NaCaH}$, and the 10,50, and 5\% ethanol fractions. The peptides derived from $\beta$-CN showed a broadly similar distribution (Figure 2). Therefore, the abundance of $\alpha_{\mathrm{S}^{-}}$and $\beta$-CN-derived peptides appears to correlate with the bitterness scores of the flash chromatography fractions (Figure 2).

In the structure of $\alpha_{\mathrm{S} 1}$-CN, 2 hydrophobic regions are separated by a polar region (Horne, 1998; Figure $3)$. These 2 hydrophobic zones contain all proline residues found in $\alpha_{\mathrm{S}^{-}} \mathrm{CN}$. When located in the center of a peptide chain, proline can increase peptide bitterness by altering the structure of the peptide, allowing it to more easily connect to a taste receptor cell (Ishibashi et al., 1988). Very few peptides originating from the hydrophilic center region of $\alpha_{S_{1}-} \mathrm{CN}$ were identified in the fractions; rather, the $\alpha_{\mathrm{S1}}-\mathrm{CN}$ peptides in all fractions originated mostly from the hydrophobic ter- 
mini (Figure 3). It appears that the $<3$-kDa permeate contained the highest abundance of peptides located on the hydrophobic $\mathrm{N}$ terminus of $\alpha_{\mathrm{S}_{1}} \mathrm{CN}$ compared with the parent $\mathrm{NaCaH}$, which did not contain many peptides from either terminus of the $\alpha_{\mathrm{S}_{1}} \mathrm{CN}$ backbone (Figure 3). The higher concentration of peptides from the $\mathrm{N}$ terminus of $\alpha_{\mathrm{S}_{1}} \mathrm{CN}$ in the $<3$-kDa permeate may explain its increased bitterness compared with the parent $\mathrm{NaCaH}$. Of the flash chromatography fractions, the most bitter fraction (30\% ethanol) contained the highest abundance of peptides originating from the hydrophobic $\mathrm{N}$ terminus of $\alpha_{\mathrm{S1}^{-}} \mathrm{CN}$, whereas peptides present in the second most bitter fraction ( $10 \%$ ethanol) were more concentrated toward the hydrophobic $\mathrm{C}$ terminus of $\alpha_{\mathrm{S}^{-}} \mathrm{CN}$ (Figure 3). Although the $30 \%$ ethanol fraction contained a higher overall abundance of $\alpha_{\mathrm{S}_{1}} \mathrm{CN}$ peptides than the $10 \%$ ethanol fraction (Figure 2), the increased abundance of peptides in the $10 \%$ ethanol fraction from the hydrophobic $\mathrm{C}$ terminus of $\alpha_{\mathrm{S} 1}-\mathrm{CN}$
(Figure 3) may have contributed to the high bitterness value of this fraction.

Of the individual caseins, $\beta$-CN is the most hydrophobic and contains the greatest number of proline residues (35), which can also influence peptide bitterness (Ishibashi et al., 1988). Although the majority of $\beta-\mathrm{CN}$ is considered to be highly hydrophobic (see Figure 4; Fox, 1982), the $\beta$-CN 193-209 region is known to contain particularly bitter peptides (Saha and Hayashi, 2001; Singh et al., 2005). Generation of bitter peptides from this region of $\beta-\mathrm{CN}$ results from chymosin action and is a major source of bitterness in cheese (Singh et al., 2005). No peptides (in any of the fractions) originated from the hydrophilic $\mathrm{N}$ terminal of $\beta$-CN. Peptides of the bitter subfractions $(<3-\mathrm{kDa}$ permeate and the 10 and $30 \%$ ethanol fractions) were concentrated in the hydrophobic dominant region of $\beta$-CN, particularly toward the $\mathrm{C}$ terminus (Figure 4). These fractions also had a high concentration of peptides overlapping in the
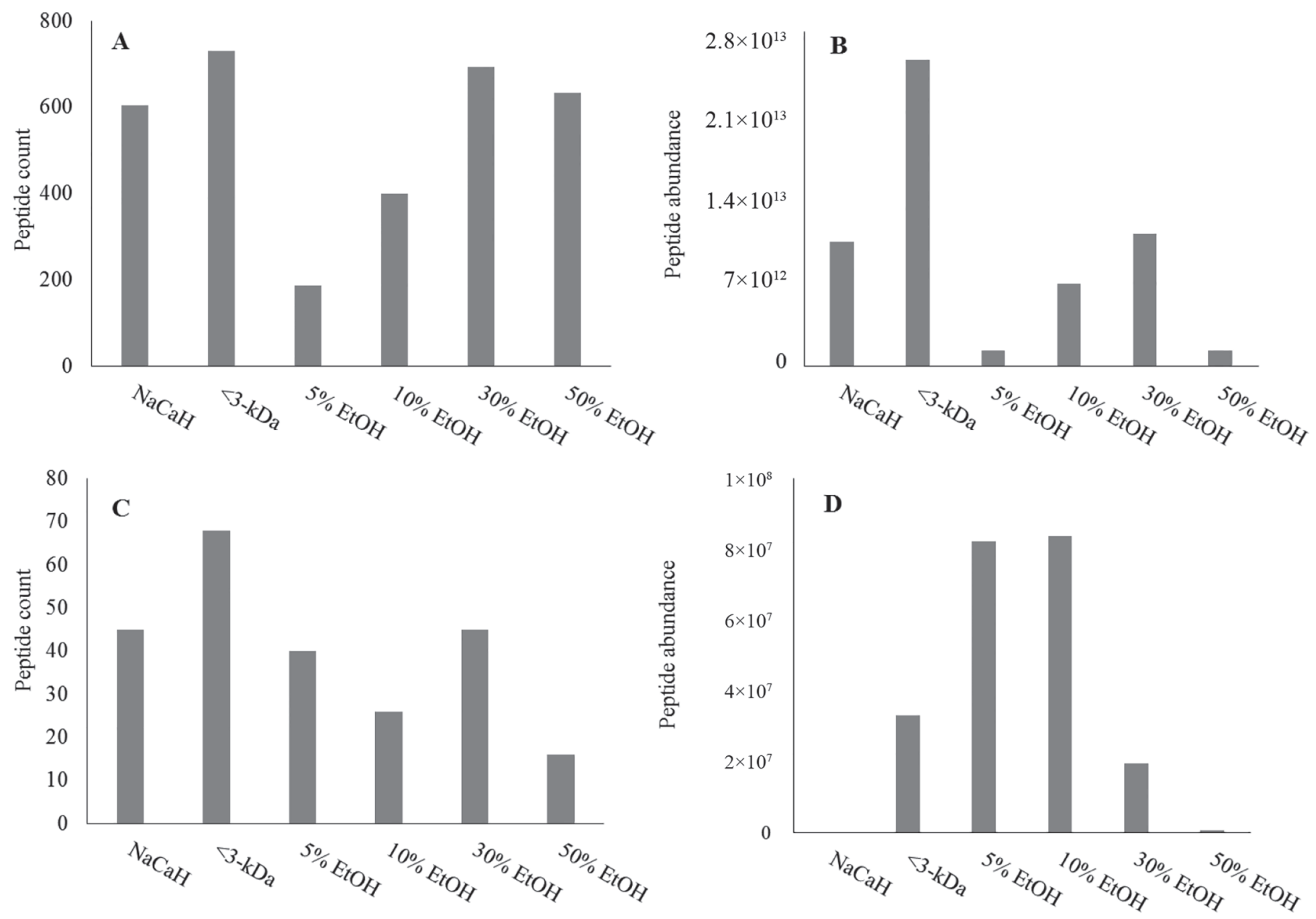

Figure 1. Unique peptide count and abundance of peptides ( $\geq 5 \mathrm{AA})$ in the sodium caseinate hydrolysate $(\mathrm{NaCaH})$, the $<3$-kDa permeate, and the flash chromatography fractions $[5,10,30$, and $50 \%$ ethanol (EtOH)] identified from (A, B) the Orbitrap MS (Thermo Fisher Scientific, Waltham, MA) data and (C, D) the quadrupole time-of-flight MS data. 


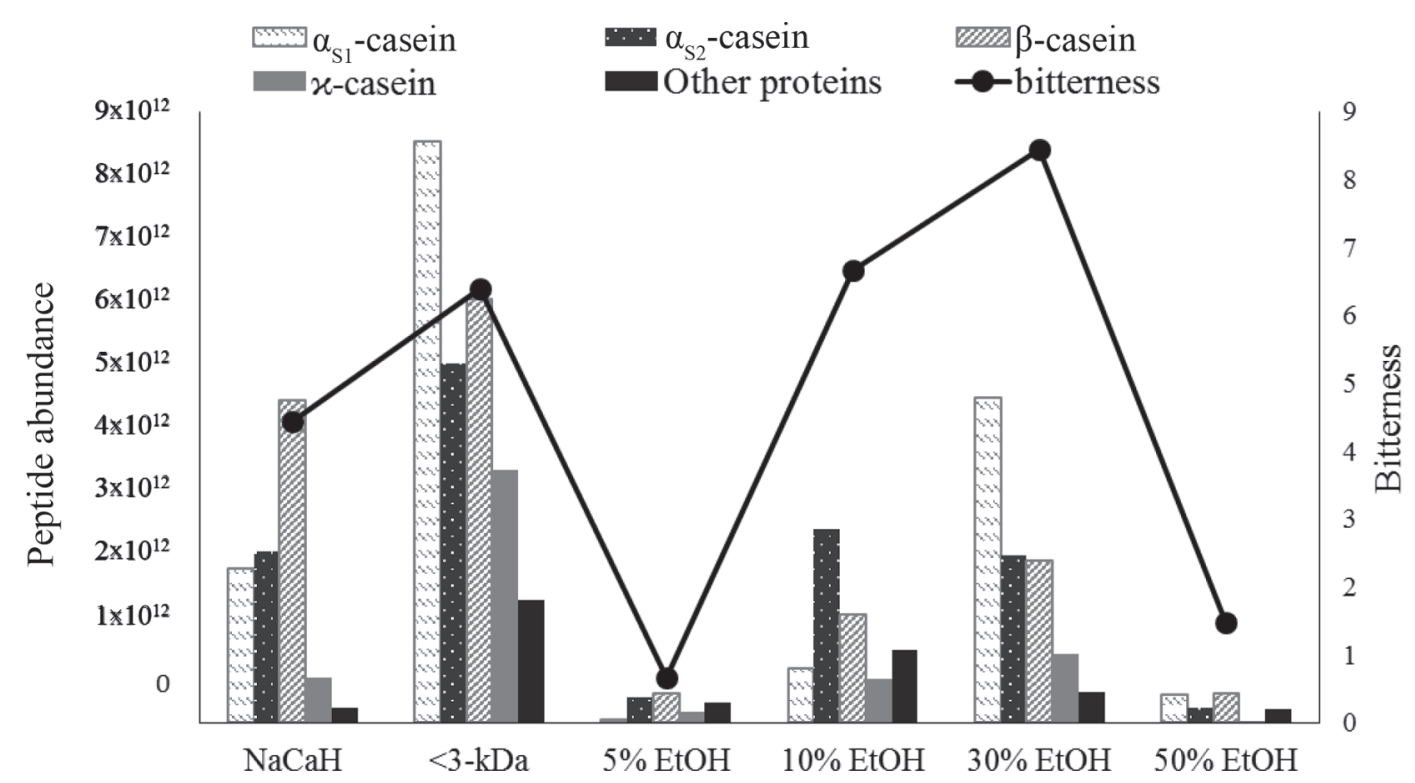

Figure 2. Abundance of peptides originating from $\alpha_{\mathrm{S}_{1}}-\mathrm{CN}, \alpha_{\mathrm{S} 2}-\mathrm{CN}, \beta-\mathrm{CN}, \kappa-\mathrm{CN}$, and other milk proteins in the sodium caseinate hydrolysate $(\mathrm{NaCaH})$, the $<3-\mathrm{kDa}$ permeate, and the flash chromatography fractions [5, 10, 30, and 50\% ethanol (EtOH)] of Orbitrap MS (Thermo Fisher Scientific, Waltham, MA) data. Bitterness of fractions is represented on the secondary axis.

bitter $\beta$-CN 193-209 region (highlighted between the black lines of Figure 4), which may have contributed to their increased bitterness. The low overall abundance of peptides derived from $\alpha_{\mathrm{S}^{-}}$and $\beta-\mathrm{CN}$ in the 5 and $50 \%$ ethanol fractions (Figure 2) and, in particular, the lack of peptides from the hydrophobic regions of these proteins (Figures 3 and 4 ) is most likely the reason for the lower bitterness scores of these fractions.

\section{Identification of Insulinogenic Bioactive Peptides}

The Orbitrap MS data revealed that the $<3-\mathrm{kDa}$ permeate contained a higher relative abundance of insulinogenic peptides $(2.8 \%$ of the total peptide abundance) than the $\mathrm{NaCaH}$, in which only $0.1 \%$ of the total peptide abundance was the insulinogenic peptides (Table 1 ). The $3-\mathrm{kDa}$ membrane filtration process likely

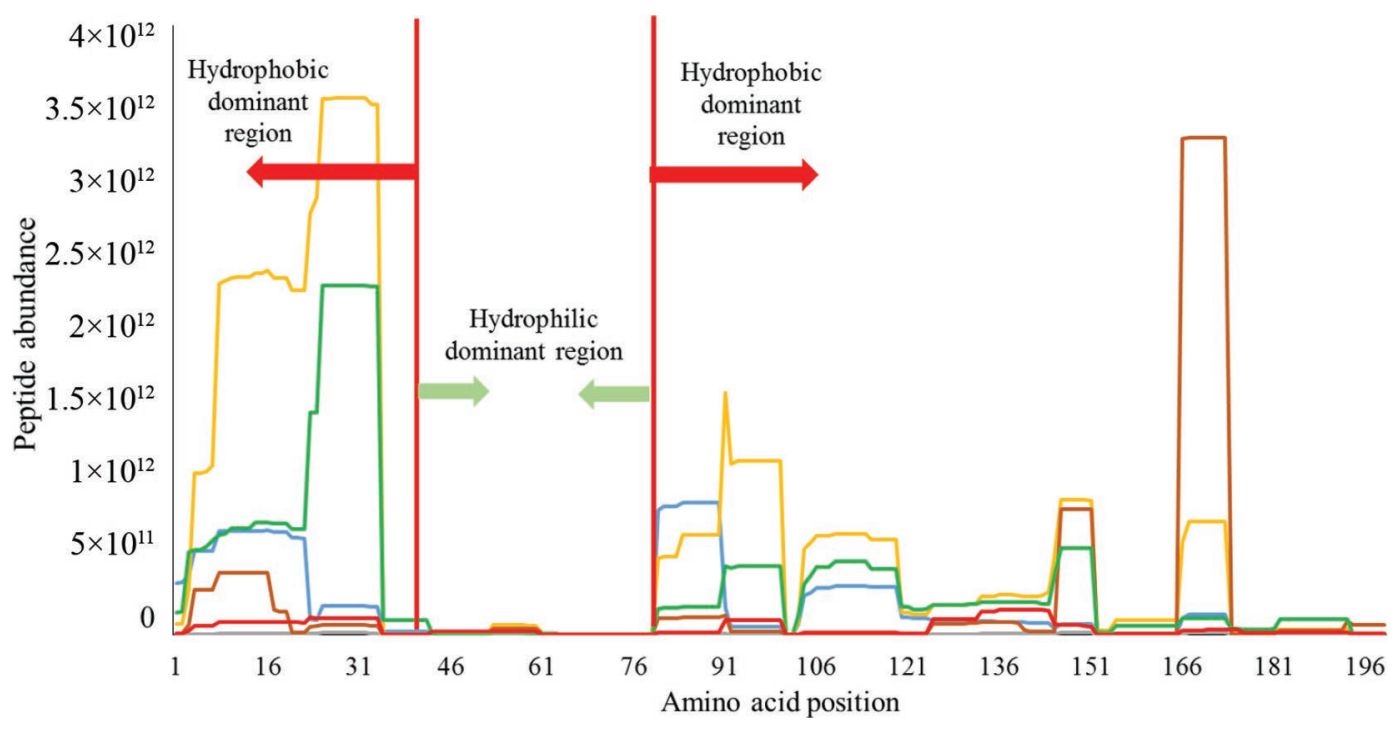

Figure 3. Abundance of peptides on the $\alpha_{\mathrm{SI}^{-}} \mathrm{CN}$ backbone (AA located on the $\mathrm{X}$-axis) for the sodium caseinate hydrolysate (blue), the $<3$ $\mathrm{kDa}$ permeate (yellow), and the flash chromatography fractions [ $5 \%$ (gray), 10\% (orange), 30\% (green), and 50\% ethanol (red)] of Orbitrap MS (Thermo Fisher Scientific, Waltham, MA) data. The area marked within the red lines represents the hydrophilic dominant region. 
eliminated (or retained) larger peptides, hence increasing the amount of insulinogenic peptides in the $<3-\mathrm{kDa}$ permeate. As membrane filtration molecular weight cut-off thresholds are approximate and multiple factors affect the process, it is unsurprising that insulinogenic peptides with molecular weights $>3 \mathrm{kDa}$ (e.g., peptide 2 in Table 1) permeated through the membrane and were identified. Within the $<3$-kDa permeate, the insulinogenic peptides were further concentrated in the most bitter flash chromatography fraction (30\% ethanol). This fraction contained the highest overall abundance of insulinogenic peptides (4\% of the total peptide abundance). The second most bitter fraction, $10 \%$ ethanol, contained a very low abundance of these peptides $(0.4 \%$ of the total peptide abundance), whereas just 0.1 and $0.3 \%$ of the total peptide abundance of the least bitter fractions, 5 and $50 \%$ ethanol, represented the insulinogenic peptides (Table 1). Although the RP-HPLC characterization of the flash chromatography fractions (Murray et al., 2017) showed good separation of the fractions, there was some overlap between the fractions, which may explain the presence of some insulinogenic peptides in the 10 and $50 \%$ ethanol fractions. The high abundance of insulinogenic peptides in the bitter $30 \%$ ethanol fraction highlights the difficulty in removing bitterness without compromising the bioactivity of this $\mathrm{NaCaH}$. The $10 \%$ ethanol fraction was the second most bitter fraction but contained a low concentration of insulinogenic peptides (compared with the $30 \%$ ethanol fraction). Therefore, removal of this fraction from the $\mathrm{NaCaH}$ could reduce bitterness without compromising bioactivity.
Ney (1979) aimed to determine the relationship between molecular weight, hydrophobicity, and bitterness of peptides. Ney (1979) demonstrated that all bitter peptides in a test set had a Q-value (calculation of a peptide chain's hydrophobicity) $>1,400 \mathrm{cal} / \mathrm{mol}$ and a molecular weight $<6 \mathrm{kDa}$, whereas nonbitter peptides had Q-values of $<1,300 \mathrm{cal} / \mathrm{mol}$ and molecular weights of $>6 \mathrm{kDa}$. The findings of some studies support Ney's Q-rule (Guigoz and Solms, 1976; Liu et al., 2014), whereas the findings of others demonstrate inconsistencies (Toelstede and Hofmann, 2008). For example, although AA position affects peptide bitterness (Ishibashi et al., 1987a, b), position is not accounted for in the model. Nevertheless, the Q-value can still be used to roughly predict whether a peptide is likely to be bitter. The Q-values of the 11 insulinogenic peptides were calculated (Table 1). The most abundant insulinogenic peptide (peptide 8 in Table 1) in the $30 \%$ ethanol fraction had a Q-value of $1,518.5 \mathrm{cal} /$ mol and a molecular weight of $<6 \mathrm{kDa}$, indicating that it is likely to be bitter according to Ney's Q-rule. An additional 4 insulinogenic peptides also had Q-values of $>1,400 \mathrm{cal} / \mathrm{mol}$, with molecular weights $<6 \mathrm{kDa}$. Four of the peptides, including the parent peptide sequence (peptide 1; Table 1), had Q-values between 1,300 and $1,400 \mathrm{cal} / \mathrm{mol}$, and therefore the bitterness of these peptides cannot be predicted with Ney's rule. Just 1 peptide had a Q-value $<1,300 \mathrm{cal} / \mathrm{mol}$, which according to Ney (1979) should not be bitter. All 11 insulinogenic peptides derive from the hydrophobic $\mathrm{C}$ terminus of $\beta-\mathrm{CN}$, known to produce bitter peptides (Singh et al., 2003).

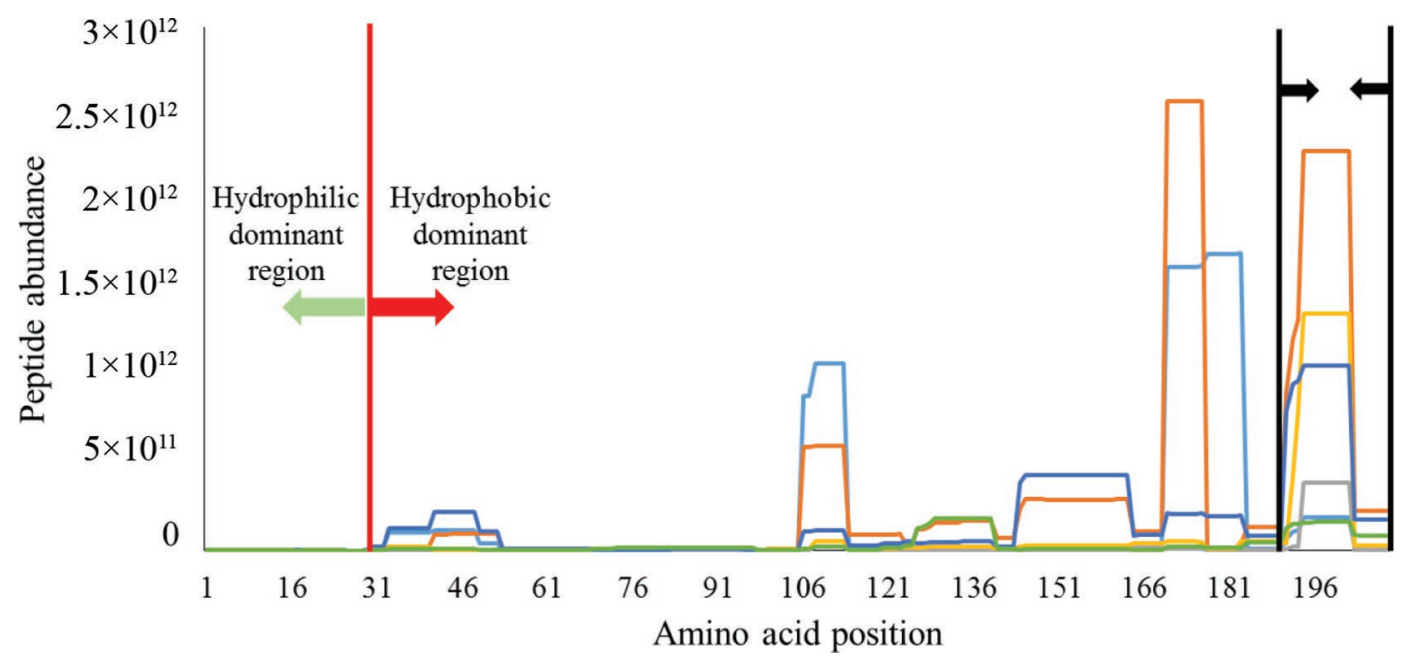

Figure 4. Abundance of peptides on the $\beta$-CN backbone (AA located on the X-axis) for the sodium caseinate hydrolysate (blue), the $<3$ $\mathrm{kDa}$ permeate (yellow), and the flash chromatography fractions [5\% (gray), 10\% (orange), 30\% (green), and 50\% ethanol (red)] of Orbitrap MS (Thermo Fisher Scientific, Waltham, MA) data. The bitter $\beta$-CN 193-209 section is marked between the 2 black lines; the area to the left of the red line is the hydrophobic dominant region. 


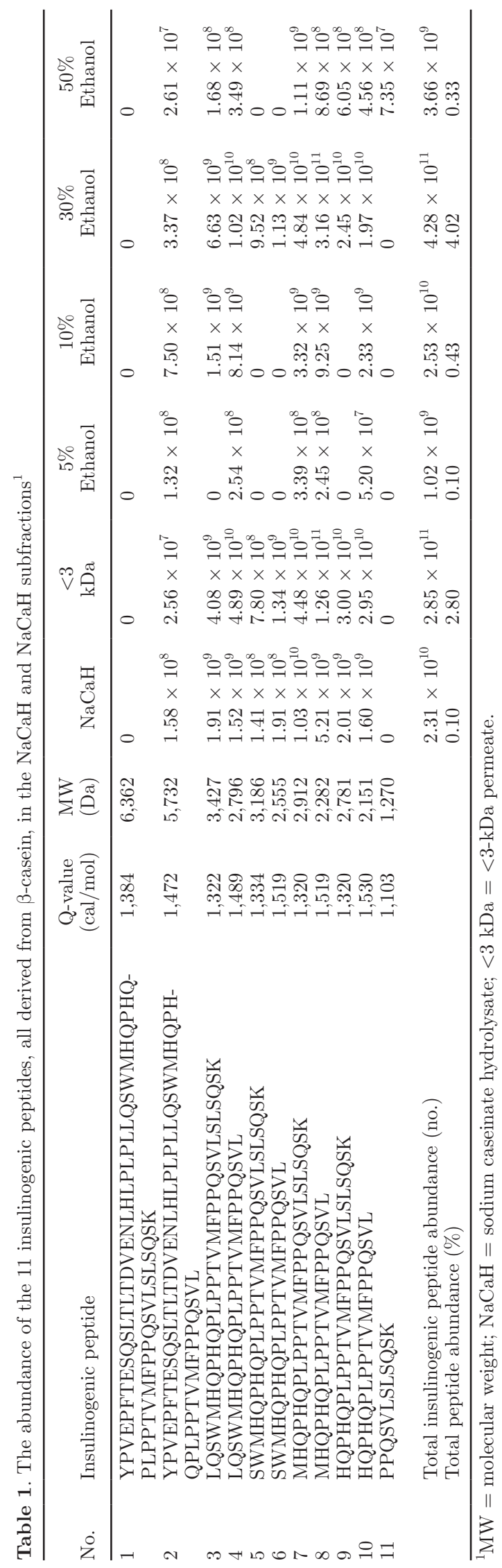

Previous studies evaluating the effect of $\mathrm{NaCaH}$ on glycemic management have been performed using relatively large doses of hydrolyzed proteins ranging from approximately 25 to $35 \mathrm{~g}$ (van Loon et al., 2003; Manders et al., 2005, 2006a,b; Jonker et al., 2011); however, more recent studies have shown that a lowered dose of $12 \mathrm{~g}$ was effective at increasing insulin concentrations in the body (Jonker et al., 2011; Brennan et al., 2015). As taste is one of the most important factors for consumer acceptance of a food product (Verbeke, 2006), consuming this amount of a bitter $\mathrm{NaCaH}$ could be a challenge and therefore hinder its potential in the market. Considering that $\mathrm{NaCaH}$ contains just $0.1 \%$ of insulinogenic peptides, it is possible that only approximately $0.012 \mathrm{~g}$ of isolated insulinogenic peptides (or $0.3 \mathrm{~g}$ of the $30 \%$ ethanol fraction) is needed to exert health benefits. Although it is not ideal for the most bitter fraction to contain the highest abundance of insulinogenic peptides, if the bioactive peptides of this $\mathrm{NaCaH}$ could be concentrated further (as they were in the $<3$-kDa permeate and $30 \%$ ethanol fraction), they could be administered at a lowered $\mathrm{NaCaH}$ dose. This would be ideal as it may be easier to mask the bitterness of a smaller, more concentrated dose of $\mathrm{NaCaH}$ in a food matrix.

\section{Identification of Literature-Identified Bioactive Peptides}

The peptides identified by Orbitrap MS were also screened for matches with literature-identified bioactive peptides (Table 2). Between 25 and 49 unique literature-identified bioactive peptides were identified in each of the $\mathrm{NaCaH}$ fractions. The biological activity of some of these peptides included ACE inhibition (24 unique peptides), antimicrobial (12), antioxidant (7), DPP-IV (6), immunomodulatory (5), opioid antagonist (3), antithrombotic (2), and caseinophosphopeptide (1) activities. The $<3$-kDa permeate contained the highest count of Orbitrap-identified bioactive peptides, followed by the $\mathrm{NaCaH}$ and the 30,50,10, and 5\% ethanol fractions (Figure 5A). Orbitrap-identified peptides known to exhibit ACE inhibition were present in the highest abundances; all other bioactivities were much less abundant (Figure 5B). The $<3$-kDa permeate contained the highest abundance of both ACE-inhibitory peptides and total bioactive peptides (Figure 5B).

There appears to be a correlation between abundance of Orbitrap-identified bioactive peptides and the bitterness of the flash chromatography fractions, with the $30 \%$ ethanol flash fraction having the highest abundance of bioactive peptides, followed by the 10,5 , and $50 \%$ ethanol fractions (Figure 5B). Although the 5 and $50 \%$ ethanol fractions contained a relatively high count 
of individual bioactive peptides (Figure 5A), the abundance of these peptides was very low (Figure 5B), which is unsurprising considering that the total abundance of peptides in these fractions was also low (Figure 1B). It is possible that the greater abundance of bioactive peptides in the $30 \%$ ethanol fraction may stem from a greater opportunity to match with literature-identified bioactive peptides rather than any concentration of bioactivity in this fraction.

An additional 10 bioactive peptides (of 2-5 AA in length) were identified in the Q-TOF MS data (5 ACE inhibitory, 1 antioxidant, 3 DPP-IV, 1 immunomodulatory, and 1 opioid agonist; Table 3). Different trends were observed within the Q-TOF MS data compared with the Orbitrap MS data. The $<3-\mathrm{kDa}$ permeate contained the highest Q-TOF-bioactive peptide count, followed by the $\mathrm{NaCaH}$ and the $5 \%$ ethanol fraction (which both contained equal peptide counts) and the 30 , 10 , and $50 \%$ ethanol fractions (Figure 6A). However, it was the $5 \%$ ethanol fraction that contained the highest abundance of the bioactive peptides (Figure 6B). The increased concentration of bioactive peptides in the $5 \%$ ethanol fraction may again stem from their reduced ability to bind to the C18 column (of the RP flash chromatography fractionation), and hence they were eluted in the most hydrophilic fraction ( $5 \%$ ethanol).

Table 2. Orbitrap ${ }^{1}$-identified bioactive peptides

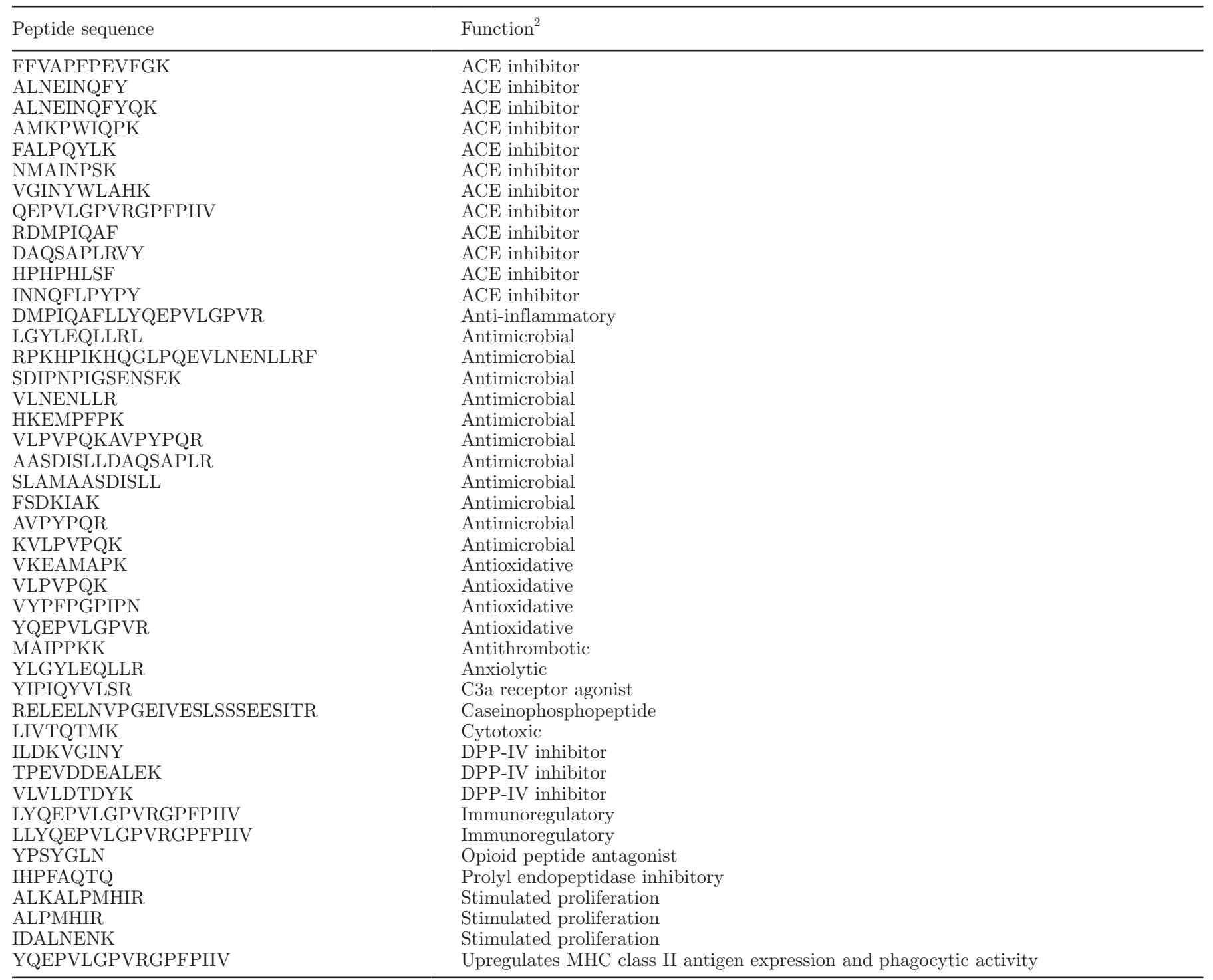

${ }^{1}$ Thermo Fisher Scientific (Waltham, MA).

${ }^{2} \mathrm{ACE}=$ angiotensin-converting enzyme; DPP-IV = dipeptidyl peptidase-4; MHC = major histocompatibility complex. 
Table 3. Quadrupole time-of-flight-identified bioactive peptides

\begin{tabular}{ll}
\hline Peptide sequence & Function $^{1}$ \\
\hline AMKPW & ACE inhibitor \\
KH & DPP-IV inhibitor \\
LL & DPP-IV inhibitor \\
LLY & Immunoregulatory \\
LQ & ACE inhibitor \\
LQSW & ACE inhibitor \\
QY & DPP-IV inhibitor \\
YLGY & ACE inhibitor \\
YLLF & ACE inhibitor, opioid agonist \\
YPEL & Antioxidative \\
\hline
\end{tabular}

$\overline{{ }^{1} \mathrm{ACE}}=$ angiotensin-converting enzyme; DPP-IV = dipeptidyl peptidase- 4 .

\section{CONCLUSIONS}

Insulinogenic peptides were enriched in the most bitter ultrafiltration subfraction (the $<3-\mathrm{kDa}$ permeate) of the $\mathrm{NaCaH}$ and were further concentrated in the most bitter flash chromatography fraction $(30 \%$ ethanol). Other literature-identified bioactive peptides, particularly peptides known to exhibit ACE-inhibitory activity, were also enriched in the $<3-\mathrm{kDa}$ permeate. Of the flash chromatography fractions, the $30 \%$ ethanol fraction also contained the highest abundance of these peptides. This finding suggests a potential correlation between bitter taste and bioactivity, highlighting the complications in removing bitterness while maintaining biological activity in the protein hydrolysate. Masking

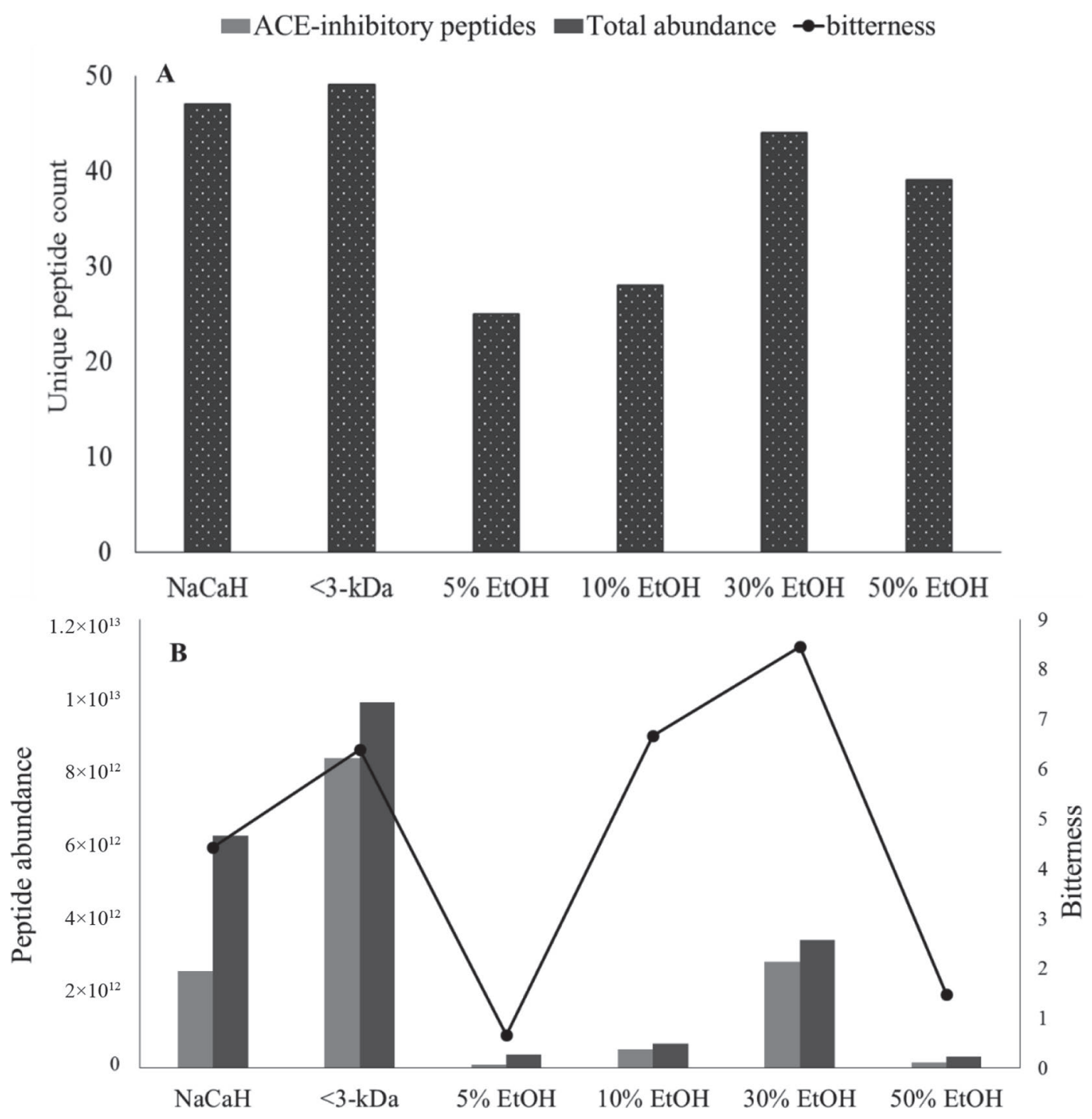

Figure 5. Unique peptide count $(\mathrm{A})$ and abundance $(\mathrm{B})$ of bioactive peptides $(\geq 5 \mathrm{AA})$ in the sodium caseinate hydrolysate (NaCaH), the $<3$-Da permeate, and the flash chromatography fractions [5, 10, 30, and 50\% ethanol (EtOH)] identified from the Orbitrap MS (Thermo Fisher Scientific, Waltham, MA) data. Bitterness of fractions is represented on the secondary axis in panel B. ACE = angiotensin-converting enzyme. 

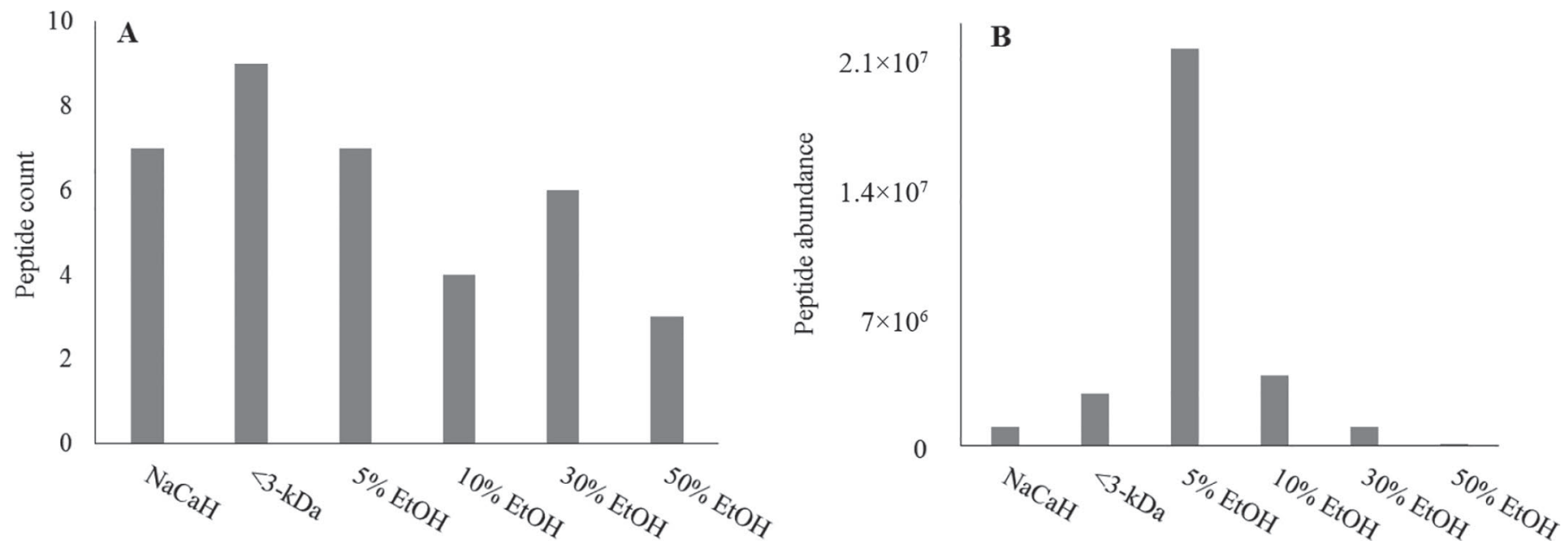

Figure 6. Unique peptide count (A) and abundance (B) of bioactive peptides ( $\leq 5 \mathrm{AA})$ in the sodium caseinate hydrolysate $(\mathrm{NaCaH})$, the $<3$ Da permeate, and the flash chromatography fractions $[5,10,30$, and 50\% ethanol (EtOH)] identified from the quadrupole time-of-flight MS data.

$\mathrm{NaCaH}$ bitterness is thus required rather than selective removal of bitter fractions in this $\mathrm{NaCaH}$. A high dose of $\mathrm{NaCaH}$ is generally required to significantly lower glucose and increase insulin in humans. However, consuming such a high amount of an $\mathrm{NaCaH}$ is an issue due to its intense bitterness. Considering that the $\mathrm{NaCaH}$ contains hundreds of peptide sequences as well as traces of fat and ash, the amount of isolated insulinogenic peptides required for biological activity may be only a few milligrams. Therefore, by isolating the most bioactive $\mathrm{NaCaH}$ fraction (potentially the $<3-\mathrm{kDa}$ permeate or $30 \%$ ethanol fraction), it may be that the dose required for the biological action could be substantially reduced, thus making bitterness masking easier. The $10 \%$ ethanol fraction contained a very low abundance of insulinogenic peptides, yet it was the second most bitter fraction. Removal of this fraction may reduce the overall bitterness of the $\mathrm{NaCaH}$ without compromising the bioactivity.

\section{ACKNOWLEDGMENTS}

The authors thank R. FitzGerald (University of Limerick, Ireland) for sharing expertise required to generate the casein hydrolysate used in this study and Brian Murray (Kerry Ingredients, Ireland) for the manufacture of the hydrolysate at Teagasc Moorepark (Cork, Ireland). We also thank Gerard Cagney and Aisling Robinson (University College Dublin, Ireland) for help with peptide mass spectrometry of the patented insulinogenic peptides in the hydrolysate. We thank the Proteomics Facility at University of California, Davis, for their assistance in in peptide identification. This work was funded by the Enterprise Ireland grant TC2013001 to Food for Health Ireland, a Fulbright-Teagasc Student
Award (Ireland), a Science Foundation Ireland Industry Fellowship grant (15/IFB/3601 to T. A. Holton), and the K99/R00 Pathway to Independence Career Award, Eunice Kennedy Shriver Institute of Child Health and Development of the National Institutes of Health (4R00HD079561, United States) to D. C. Dallas (Oregon State University, Corvallis, OR).

\section{REFERENCES}

Behrens, M., and W. Meyerhof. 2011. Gustatory and extragustatory functions of mammalian taste receptors. Physiol. Behav. 105:4-13. Brennan, L., A. Robinson, N. Noronha, D. Fitzgerald, A. Nongonierma, T. Holton, H. Roche, J. Jacquier, D. Shields, and E. Gibney. 2015. Peptides and compositions thereof for improvement of glycaemic management in a mammal. Ireland pat. no. WO2015173266 A1.

Chatchatee, P., K. M. Järvinen, L. Bardina, L. Vila, K. Beyer, and H. Sampson. 2001. Identification of IgE and IgG binding epitopes on $\beta$ - and $\kappa$-casein in cow's milk allergic patients. Clin. Exp. Allergy 31:1256-1262.

Ciborowski, P., and J. Silberring. 2016. Proteomic Profiling and Analytical Chemistry: The Crossroads. Elsevier, Amsterdam, the Netherlands.

Craig, R., and R. C. Beavis. 2004. TANDEM: Matching proteins with tandem mass spectra. Bioinformatics 20:1466-1467.

Dallas, D. C., F. Citerne, T. Tian, V. L. Silva, K. M. Kalanetra, S. A. Frese, R. C. Robinson, D. A. Mills, and D. Barile. 2016. Peptidomic analysis reveals proteolytic activity of kefir microorganisms on bovine milk proteins. Food Chem. 197:273-284.

Dallas, D. C., A. Guerrero, N. Khaldi, P. A. Castillo, W. F. Martin, J. T. Smilowitz, C. L. Bevins, D. Barile, J. B. German, and C. B. Lebrilla. 2013. Extensive in vivo human milk peptidomics reveals specific proteolysis yielding protective antimicrobial peptides. J. Proteome Res. 12:2295-2304.

Dallas, D. C., A. Guerrero, E. A. Parker, L. A. Garay, A. Bhandari, C. B. Lebrilla, D. Barile, and J. B. German. 2014. Peptidomic profile of milk of Holstein cows at peak lactation. J. Agric. Food Chem. 62:58-65.

Fox, P. F. 1982. Developments in Dairy Chemistry. 4: Functional Milk Proteins. Elsevier, Amsterdam, the Netherlands.

Guigoz, Y., and J. Solms. 1976. Bitter peptides, occurrence and structure. Chem. Senses 2:71-84. 
Hernández-Ledesma, B., M. J. García-Nebot, S. Fernández-Tomé, L. Amigo, and I. Recio. 2014. Dairy protein hydrolysates: Peptides for health benefits. Int. Dairy J. 38:82-100.

Holton, T. A., V. Vijayakumar, D. C. Dallas, A. S. Guerrero, R. A. Borghese, C. B. Lebrilla, J. B. German, D. Barile, M. A. Underwood, and D. C. Shields. 2014. Following the digestion of milk proteins from mother to baby. J. Proteome Res. 13:5777-5783.

Horne, D. S. 1998. Casein interactions: Casting light on the black boxes, the structure in dairy products. Int. Dairy J. 8:171-177.

Irshad, I., A. Kanekanian, A. Peters, and T. Masud. 2015. Antioxidant activity of bioactive peptides derived from bovine casein hydrolysate fractions. J. Food Sci. Technol. 52:231-239.

Ishibashi, N., Y. Arita, H. Kanehisa, K. Kouge, H. Okai, and H. Fukui. 1987a. Bitterness of leucine-containing peptides. Agric. Biol. Chem. 51:2389-2394.

Ishibashi, N., T. Kubo, M. Chino, H. Fukui, I. Shinoda, E. Kikuchi, H. Okai, and S. Fukui. 1988. Taste of proline-containing peptides. Agric. Biol. Chem. 52:95-98.

Ishibashi, N., K. Sadamori, O. Yamamoto, H. Kanehisa, K. Kouge, E. Kikuchi, H. Okai, and H. Fukui. 1987b. Bitterness of phenylalanine- and tyrosine-containing peptides. Agric. Biol. Chem. 51:3309-3313.

Iwaniak, A., P. Minkiewicz, M. Darewicz, K. Sieniawski, and P. Starowicz. 2016. BIOPEP database of sensory peptides and amino acids. Food Res. Int. 85:155-161.

Jonker, J. T., M. Wijngaarden, J. Kloek, Y. Groeneveld, C. Gerhardt, R. Brand, A. Kies, J. Romijn, and J. Smit. 2011. Effects of low doses of casein hydrolysate on post-challenge glucose and insulin levels. Eur. J. Intern. Med. 22:245-248.

Karametsi, K., S. Kokkinidou, I. Ronningen, and D. G. Peterson. 2014. Identification of bitter peptides in aged cheddar cheese. J. Agric. Food Chem. 62:8034-8041.

Leksrisompong, P. P., R. E. Miracle, and M. Drake. 2010. Characterization of flavor of whey protein hydrolysates. J. Agric. Food Chem. 58:6318-6327.

Liu, X., D. Jiang, and D. G. Peterson. 2014. Identification of bitter peptides in whey protein hydrolysates. J. Agric. Food Chem. 62:5719-5725.

Ma, B., K. Zhang, C. Hendrie, C. Liang, M. Li, A. Doherty-Kirby, and G. Lajoie. 2003. PEAKS: Powerful software for peptide de novo sequencing by tandem mass spectrometry. Rapid Commun. Mass Spectrom. 17:2337-2342.

Manders, R. J., R. Koopman, W. E. Sluijsmans, R. van den Berg, K. Verbeek, W. H. Saris, A. J. Wagenmakers, and L. J. van Loon. 2006a. Co-ingestion of a protein hydrolysate with or without additional leucine effectively reduces postprandial blood glucose excursions in Type 2 diabetic men. J. Nutr. 136:1294-1299.

Manders, R. J., S. F. Praet, R. C. Meex, R. Koopman, A. L. de Roos, A. J. Wagenmakers, W. H. Saris, and L. J. van Loon. 2006b. Protein hydrolysate/leucine co-ingestion reduces the prevalence of hyperglycemia in type 2 diabetic patients. Diabetes Care 29:27212722.

Manders, R. J., A. J. Wagenmakers, R. Koopman, A. H. G. Zorenc, P. Menheere, N. C. Schaper, W. H. Saris, and L. J. van Loon. 2005. Co-ingestion of a protein hydrolysate and amino acid mixture with carbohydrate improves plasma glucose disposal in patients with type 2 diabetes. Am. J. Clin. Nutr. 82:76-83.

Minkiewicz, P., J. Dziuba, A. Iwaniak, M. Dziuba, and M. Darewicz. 2008. BIOPEP database and other programs for processing bioactive peptide sequences. J. AOAC Int. 91:965-980.

Murray, N. M., D. O'Riordan, J. C. Jacquier, M. O'Sullivan, J. L. Cohen, H. Heymann, D. Barile, and D. C. Dallas. 2017. Validation of a paper-disk approach to facilitate the sensory evaluation of bitterness in dairy protein hydrolysates from a newly developed food-grade fractionation system. J. Sens. Stud. https://doi.org/10 $.1111 /$ joss. 12266 .

Newman, J., T. Egan, N. Harbourne, D. O'Riordan, J. C. Jacquier, and M. O'Sullivan. 2014. Correlation of sensory bitterness in dairy protein hydrolysates: Comparison of prediction models built using sensory, chromatographic and electronic tongue data. Talanta 126:46-53.

Ney, K. H. 1979. Bitterness of peptides: Amino acid composition and chain length. Pages 149-173 in Food Taste Chemistry. J. C. Boudreau, ed. American Chemical Society, Washington, DC.

Nielsen, S. D., R. L. Beverly, Y. Qu, and D. C. Dallas. 2017. Milk bioactive peptide database: A comprehensive database of milk protein-derived bioactive peptides and novel visualization. Food Chem. 232:673-682.

Nongonierma, A. B., and R. J. FitzGerald. 2015. The scientific evidence for the role of milk protein-derived bioactive peptides in humans: A review. J. Funct. Foods 17:640-656.

Nongonierma, A. B., and R. J. FitzGerald. 2017. Strategies for the discovery and identification of food protein-derived biologically active peptides. Trends Food Sci. Technol. 69:289-305.

O'Sullivan, D., A. B. Nongonierma, and R. J. FitzGerald. 2017. Bitterness in sodium caseinate hydrolysates: Role of enzyme preparation and degree of hydrolysis. J. Sci. Food Agric. 97:4652-4655.

Power, O., P. Jakeman, and R. J. FitzGerald. 2013. Antioxidative peptides: Enzymatic production, in vitro and in vivo antioxidant activity and potential application of milk-derived antioxidative peptides. Amino Acids 44:797-820.

Regard, J. B., H. Kataoka, D. A. Cano, E. Camerer, L. Yin, Y.-W. Zheng, T. S. Scanlan, M. Hebrok, and S. R. Coughlin. 2007. Probing cell type-specific functions of Gi in vivo identifies GPCR regulators of insulin secretion. J. Clin. Invest. 117:4034-4043.

Saavedra, L., E. M. Hebert, C. Minahk, and P. Ferranti. 2013. An overview of "omic" analytical methods applied in bioactive peptide studies. Food Res. Int. 54:925-934.

Saha, B. C., and K. Hayashi. 2001. Debittering of protein hydrolyzates. Biotechnol. Adv. 19:355-370.

Schilling, B., M. J. Rardin, B. X. MacLean, A. M. Zawadzka, B. E. Frewen, M. P. Cusack, D. J. Sorensen, M. S. Bereman, E. Jing, C. C. Wu, E. Verdin, C. Ronald Kahn, M. J. MacCoss, and B. W. Gibson. 2012. Platform independent and label-free quantitation of proteomic data using MS1 extracted ion chromatograms in Skyline. Application to protein acetylation and phosphorylation. Mol. Cell. Proteomics 11:202-214.

Singh, T., M. Drake, and K. Cadwallader. 2003. Flavor of cheddar cheese: A chemical and sensory perspective. Compr. Rev. Food Sci. Food Saf. 2:166-189.

Singh, T. K., N. D. Young, M. Drake, and K. R. Cadwallader. 2005. Production and sensory characterization of a bitter peptide from $\beta$-casein. J. Agric. Food Chem. 53:1185-1189.

Toelstede, S., and T. Hofmann. 2008. Sensomics mapping and identification of the key bitter metabolites in Gouda cheese. J. Agric. Food Chem. 56:2795-2804.

Udenigwe, C. C. 2014. Bioinformatics approaches, prospects and challenges of food bioactive peptide research. Trends Food Sci. Technol. 36:137-143.

van Loon, L. J., M. Kruijshoop, P. P. Menheere, A. J. Wagenmakers, W. H. Saris, and H. A. Keizer. 2003. Amino acid ingestion strongly enhances insulin secretion in patients with long-term type 2 diabetes. Diabetes Care 26:625-630.

Verbeke, W. 2006. Functional foods: Consumer willingness to compromise on taste for health? Food Qual. Prefer. 17:126-131. 